\title{
Dermatology
}

\section{Spironolactone for the Treatment of Acne: A 4-Year Retrospective Study}

\author{
Radhika Grandhi Ali Alikhan \\ Department of Dermatology, University of Cincinnati, Cincinnati, OH, USA
}

\section{Keywords}

Acne $\cdot$ Female $\cdot$ Postadolescent acne

\begin{abstract}
Prior studies have demonstrated that spironolactone is an effective second-line treatment option for postadolescent acne, but has notable side effects. Data are, however, limited. We therefore present a 4-year retrospective study evaluating 291.5 patient-years of spironolactone for the treatment of acne. Our results showed that $86 \%$ of patients improved on spironolactone therapy. Further, patients who improved showed minimal side effects, supporting recent evidence that spironolactone is a safe option for acne treatment with a low risk of short-term adverse effects such as hyperkalemia. It is suggested that our study encourages consideration of spironolactone for postadolescent acne.
\end{abstract}

(c) 2017 S. Karger AG, Basel

\section{Introduction}

Acne affects more than 50 million Americans annually [1], and the proportion of postadolescents with acne has increased over the last decade. Postadolescent acne primarily affects females and is resistant to conventional treatment in 79-82\% of cases [2]. Spironolactone is used as a second-line treatment in this population, with use primarily limited due to its side-effect profile [3]. In this 4-year retrospective study at a large academic center, we aim to characterize the efficacy and tolerability of spironolactone for the treatment of acne.

\section{Materials and Methods}

For further details, see the online supplementary materials (see www.karger.com/doi/10.1159/000471799 for all online suppl. material) $[4,5]$ (Fig. 1-3; Tables 1, 2).

\section{Discussion}

Our findings regarding the efficacy and safety of combination therapy are consistent with those described in smaller studies $[2,4,6-8]$. The vast majority of our patients improved on spironolactone, despite many previously failing other acne treatments. Furthermore, relatively low doses were needed compared to those used for hirsutism and androgenetic alopecia [4].

\section{KARGER}

E-Mail karger@karger.com

www.karger.com/drm
(C) 2017 S. Karger AG, Basel

Radhika Grandhi, MD

Department of Dermatology, University of Cincinnati PO Box 670592, 231 Albert Sabin Way, ML \#0592

Cincinnati, OH 45267-0592 (USA)

E-Mail grandhrr@mail.uc.edu 
Fig. 1. Flowchart of Materials and Methods: cohort selection. In total, 1,566 patients were identified in our query of patients who were on spironolactone between July 17, 2012, and February 16, 2016. Of these patients, only 400 patients had never used spironolactone previously and had at least 1 note with an acne status (improved, worsened, unchanged, indeterminate) detailed in the note.

Fig. 2. Treatment response based on treatment regimen. Treatment response of 400 patients on different treatment regimens including spironolactone only, spironolactone plus oral treatment, spironolactone plus topical treatment, and spironolactone, oral treatment, and topical treatment. The $x$-axis shows the acne status posttreatment. The graph illustrates that the majority of patients were in the "improved" category, and the treatment regimen most commonly used for "improved" outcomes was combination therapy: oral therapy, topical therapy, and spironolactone.
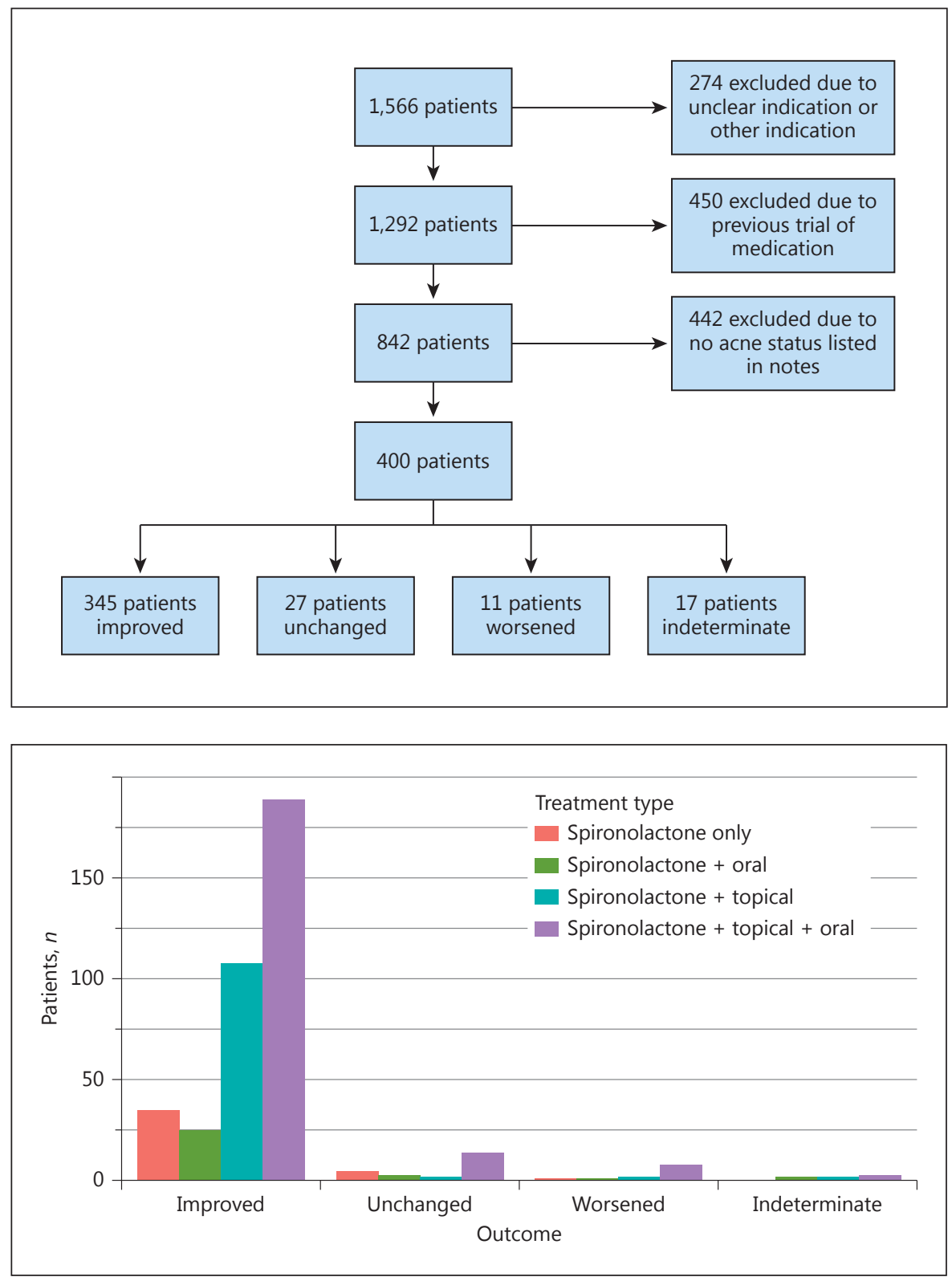

Table 1 and Figure 2 demonstrate that spironolactone is efficacious when used in combination with other agents - combining agents with different mechanisms of action in the treatment of acne is recommended by the American Academy of Dermatology [7, 9]. Table 2 demonstrates that side effects were uncommon and less frequent than previously reported, particularly headaches and menstrual irregularities $[2,4]$. Interestingly, while studies reported that $17.5-18 \%$ of patients on $50-100 \mathrm{mg}$ of spironolactone experienced menstrual irregularities $[2,4]$, only 1 patient $(0.25 \%)$ experienced these in our study. This may have been partially due to a larger per- centage of patients in our study on hormonal therapy (34.5 vs. 12\%) [4]. The frequency of xerosis in our study is consistent with that in other studies [4]. Furthermore, all patients who experienced xerosis were applying tretinoin, which can also cause xerosis [6]. The infrequent side effect of hyperkalemia, with the majority being mild, is consistent with that reported by Plovanich et al. [3]. In our short-term study, there were no patients who developed estrogen-dependent cancers, consistent with previous data published by Biggar et al. [10].

There are several limitations to this study. The major limitations are the approach, the short follow-up time for 
Fig. 3. Comparing treatment outcome for those previously treated for acne vs. those who were initially started on spironolactone. Of the 253 patients who had been previously treated for acne, after starting spironolactone $220(86.96 \%)$ improved, 4 (1.58\%) had an indeterminate acne status, $17(6.72 \%)$ were unchanged, and $12(4.74 \%)$ worsened. The remaining 147 (36.75\%) patients were untreated prior to initiating spironolactone. Of these, 137 (93.2\%) improved, $3(2.04 \%)$ had an indeterminate status, $7(4.76 \%)$ were unchanged, and 0 worsened.

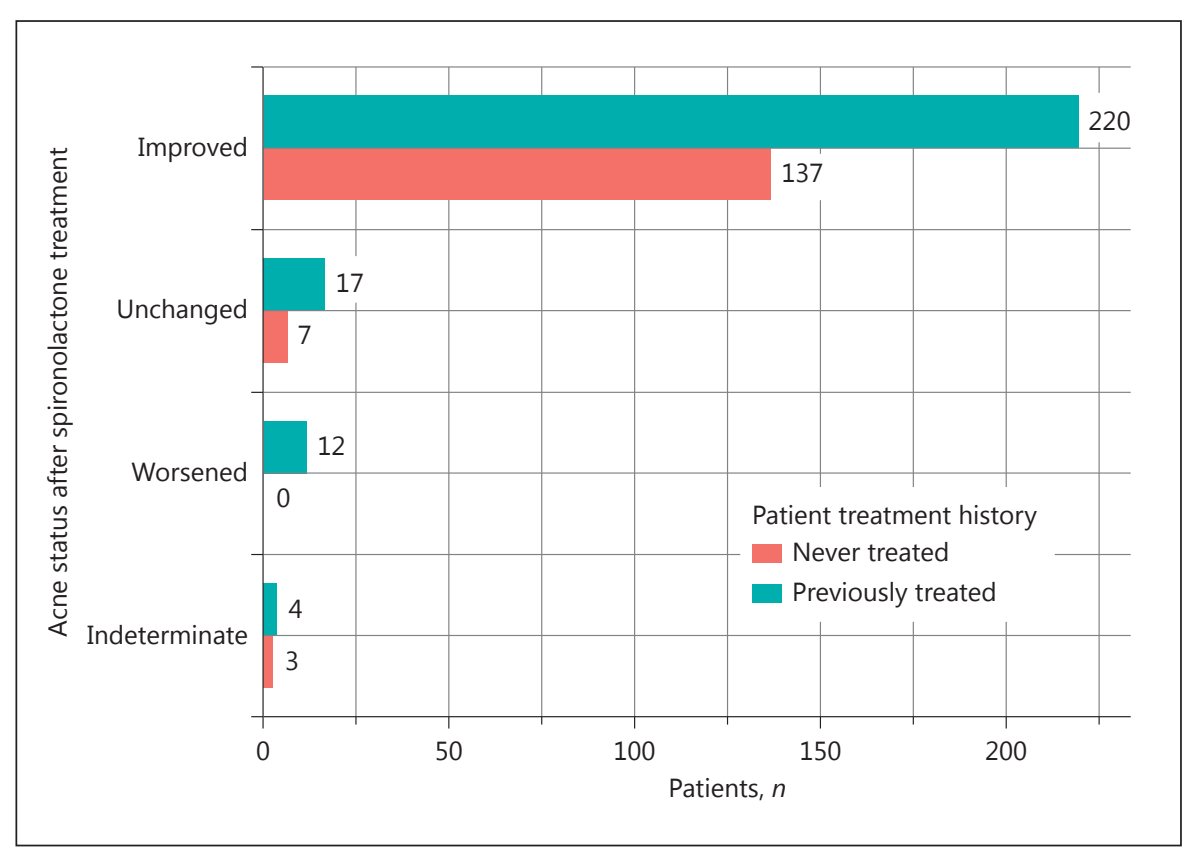

Table 1. Treatment regimen and response to treatment

\begin{tabular}{lrr}
\hline & $n$ & $\%$ \\
\hline Spironolactone + topical + oral & 189 & 88 \\
Improved & 3 & 1 \\
Indeterminate & 14 & 7 \\
Unchanged & 8 & 4 \\
Worsened & 214 & \\
\hline Total & & \\
\hline Spironolactone + oral & 25 & 81 \\
Improved & 2 & 6 \\
Indeterminate & 3 & 10 \\
Unchanged & 1 & 3 \\
Worsened & 31 & \\
Total & & \\
\hline Spironolactone only & 35 & 85 \\
Improved & 0 & 0 \\
Indeterminate & 5 \\
Unchanged & 12 \\
Worsened & 1 & 2 \\
Total & 41 & \\
\hline Spironolactone + topical & & \\
Improved & \\
Indeterminate & 108 & 95 \\
Unchanged & 2 & 2 \\
Worsened & 2 & 2 \\
Total & 2 & 2 \\
\hline
\end{tabular}

Of the total of 400 patients involved, 214 (53.5\%) used a combination of spironolactone, topical agent(s), and an oral treatment; 31 (7.75\%) used spironolactone with other oral treatment(s); 114 (28.5\%) used spironolactone with topical treatment(s); and 41 (10.25\%) used spironolactone monotherapy.

Spironolactone for the Treatment of Acne
Table 2. Side effects

Estrogen-dependent cancers $\quad 0$

Breast tenderness 1

Abdominal fullness $\quad 1$

Back pain 1

Heavy menstrual cycles $\quad 1$

Feeling "sick" 1

Severe headaches 1

Facial/perioral xerosis, erythema,

irritation, and pruritus $\quad 4$

Hyperkalemia 6

many patients, and the choice of outcomes. While the physician's routine clinical assessment of acne severity reflects the practical real-world results, it is, nevertheless, physician dependent. This study describes our experience of using spironolactone in the management of acne. The data suggest that it is a useful drug, and the results further support that spironolactone is a safe medication with tolerable side effects. It may be speculated that an increased use of spironolactone may decrease our dependence on oral antibiotics in acne management, which is paramount in this age of antibiotic stewardship [9].

\section{Key Message}

Spironolactone is a safe medication for postadolescent female acne.

Dermatology 2017;233:141-144

DOI: $10.1159 / 000471799$ 


\section{Statement of Ethics}

The study protocol was approved by the University of Cincinnati Institutional Review Board.

\section{Disclosure Statement}

The authors of this paper have no conflicts of interest to disclose.

\section{References}

1 Bhate K, Williams HC: Epidemiology of acne vulgaris. Br J Dermatol 2013;168:474-485.

2 Kim GK, Del-Rosso JQ: Oral spironolactone in post-teenage female patients with acne vulgaris: practical considerations for the clinical based on current data and clinical experience. J Clin Aesthet Dermatol 2012;5:37-50.

3 Plovanich M, Weng QY, Mostaghimi A: Low usefulness of potassium monitoring among healthy young women taking spironolactone for acne. JAMA Dermatol 2015;151:941-944.

4 Shaw JC: Low-dose adjunctive spironolactone in the treatment of acne in women: a retrospective analysis of 85 consecutively treated patients. J Am Acad Dermatol 2000;43:498502.
5 Katsambas AD, Dessinioti C: Hormonal therapy for acne: why not as first line therapy? Facts and controversies. Clin Dermatol 2010; 28:17-23.

6 Yin NC, McMichael AJ: Acne in patients with skin of color: practical management. Am J Clin Dermatol 2014;15:7-16.

7 Turoskwi CB, James WD: The efficacy and safety of amoxicillin, trimethoprim-sulfamethoxazole, and spironolactone for treatment-resistant acne vulgaris. Adv Dermatol 2007;23:155-163.
8 Dreno B, Thiboutot D, Gollnick H, Bettoli V, Kang S, et al: Antibiotic stewardship in dermatology: limiting antibiotic use in acne. Eur J Dermatol 2014;24:330-334.

9 Zaenglein AL, Pathy AL, Schlosser BJ, Alikhan A, Baldwin HE, et al: Guidelines of care for the management of acne vulgaris. J Am Acad Dermatol 2016;74:1-62.

10 Biggar RJ, Andersen EW, Wohlfahrt J, Melbye $\mathrm{M}$ : Spironolactone use and the risk of breast and gynecologic cancers. Cancer Epidemiol 2013;37:870-875. 